\title{
FPGA and ASIC Implementation and Testing of IR- UWB Baseband transceiver for IEEE802.15.4a
}

\author{
Sonom Olonbayar \\ IHP \\ Frankfurt Oder, Germany \\ sonom@ihp-microelectronics.com
}

\author{
Dan Kreiser, Rolf Kraemer \\ IHP \\ Frankfurt Oder, Germany \\ kreiser@ihp-microlectronics.com
}

\begin{abstract}
A digital baseband was designed and implemented according to the standard IEEE802.15.4a both in FPGA and as well as ASIC. The baseband supports data rates $850 \mathrm{~Kb} / \mathrm{s}, 6.81$ $\mathrm{Mb} / \mathrm{s}$ and $27.24 \mathrm{Mb} / \mathrm{s}$ running at the clock speed of 31.2 MHz. The transmitter and receiver were tested by introducing various distortions to the signal being received. The baseband was shown to be fully functional being able to receive even under heavy distortion. Both the synchronization and data detection performance are robust. The baseband tested with a FPGA was further made as an ASIC in the $250 \mathrm{~nm}$ BiCMOS technology from IHP, Germany.
\end{abstract}

Index Terms-IEEE802.15.4a, Synchronization, detection, SNR, distortion, error rate, digital waveform, packet structure

\section{INTRODUCTION}

IR-UWB is based on transmitting short pulses that carry data bits. It operates in the frequency band of 3.1-10.6 GHz. The standard IEEE802.15.4a specifies IR-UWB for short range wireless communications to support low to medium data rates. As the bandwidth of IR-UWB is large being higher than 500 $\mathrm{MHz}$ and the emission level is as low as $-41.3 \mathrm{dBm} / \mathrm{MHz}$, it presents challenge in implementing it in hardware available today. There is a number of research works dealing with implementation of IR-UWB and they mostly consider part of the system. This paper discusses a complete IR-UWB transceiver realized in FPGA as well as ASIC. This baseband was made as a part of a fully integrated solution comprising high frequency frontend in the $250 \mathrm{~nm}$ BiCMOS process at IHP, Germany. This publication concerns not only pure implementation but it also provides theoretical considerations where necessary.

\section{IR-UWB TRANSMITTER}

According to the standard stated, the baseband can support data rates $850 \mathrm{~Kb} / \mathrm{s}, 6.81 \mathrm{Mb} / \mathrm{s}$ and $27.21 \mathrm{Mb} / \mathrm{s}$. The transmitter was built to be configured in a number of ways. Before starting to transmit, it is set to the mode needed. The modes can be various data rates and preamble length, turning on and off transmitter and receiver with and without channel coding and decoding and certain test modes. All these settings are sent from the medium access (MAC) layer to the baseband via a SPI interface integrated. After that the data to be sent is written to the memory contained in the baseband. The memory is realized in the form of FIFO having the capacity of 154 Bytes. Once the transmitter is set to a certain mode and the memory
RAM is loaded with data by MAC, the data can be sent with a further instruction. Main operations that take place at the transmitter are channel encoding with convolutional and ReedSolomon coders, Binary Position Modulation (BPM) and Binary Phase Shift Keying (BPSK) and linear phase shift register (LFSR). The block diagram of the baseband transmitter is shown in Figure 1.

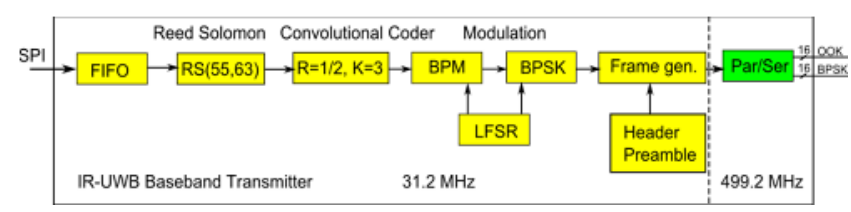

Figure 1: Block diagram of IR-UWB baseband transmitter

As can be seen all algorithms till parallel to serial conversion (Par/Ser) are implemented with the clock $31.25 \mathrm{MHz}$. It was chosen due its power efficiency, possibility to test the baseband with commercially available FPGAs and suitability to be realized as ASIC in $250 \mathrm{~nm}$ BiCMOS process. Systematic Reed-Solomon encoding $\operatorname{RS}_{6}(63,55)$ is applied to counter with the errors that can occur in bursts during the transmission. It adds 48 bits (6 symbols) to every block of data having 330 bits which is converted to 55 symbols. Systematic Reed Solomon encoded data is then encoded with the half rate systematic convolutional coder built from the polynomial $g O=[010]_{2}$, $g l=[101]_{2}$. As both encoders are systematic, they produce the input data unaltered at its output followed by some redundant bits introduced by them. Therefore it is possible to turn off the decoders individually still receiving data completely without correction.

BPM was implemented by placing a burst within symbol duration either at the first or second half which represents bits " 0 " or " 1 " respectively. The symbol duration varies according to the data rate to be used. For $850 \mathrm{~Kb} / \mathrm{s}$, it is $1024 \mathrm{~ns}$ and the burst length is 16 chips (pulses) occupying 32 ns. The bursts are made from positive as well as negative pulses. Bursts are generated from a LFSR based on the generator polynomial $s_{n}=s_{n-14} \oplus s_{n-15}$ which does XOR for the outputs of $14^{\text {th }}$ and $15^{\text {th }}$ and feeds the results to the input. It produces 16 chips for each cycle of the clock $31.2 \mathrm{MHz}$. 
The implementation adopted in this publication focuses on inserting 15 zeros between the elements of the pseudo code used in preamble. The standard also foresees to insert 63 zeros which are not reflected in this work. After a frame is generated at the rate $31.25 \mathrm{MHz}$, it is serialized with the clock 499.2 $\mathrm{MHz}$.

For the data rate of $850 \mathrm{~Kb} / \mathrm{s}$, there is a guard interval of $256 \mathrm{~ns}$ between two adjacent burst. The burst position varies for every symbol taking values one to eight. In case of $6.81 \mathrm{Mb} / \mathrm{s}$ and $27.21 \mathrm{Mb} / \mathrm{s}$, the symbol duration reduces to $256 \mathrm{~ns}$ and $128 \mathrm{~ns}$ respectively. The number of pulses making up bursts is also shortened to 2 and 1 . As a consequence the guard interval becomes smaller. Hence with increasing data rates, the receiver has to detect fewer pulses even single pulse.

BPSK is done by shifting the phase of individual pulses of bursts by $180^{\circ}$ degrees which carries polarity bits implying that two bits are sent within a symbol. The task of the receiver is not only to detect presence of bursts which is referred as energy detection but also their phase shift. Once data bits are encoded completely the transmitter generates a frame prepending a header to it. Header is built from three different parts: Synchronization header SHR, Start of Frame Delimeter (SFD) and PHY header (PHR). SHR consists of ternary codes $(1,0,-1)$ containing positive, negative and zero positions repeated 16, 64, 1024 or 4096 times [2] (See Figure 2). Figure 2 corresponds to the frame where bits are represented by symbols each of which carrying two bits. The receiver synchronizes to the SHR (preamble) and the gain is set to the proper value within this period. SFD indicates the end of header and the start of the data field. PHR carries useful information that is necessary to detect data bits such as data rate, frame length etc. The 13 bits length PHR is double protected: firstly with a Hamming encoding extending to 19 bits and secondly the half rate convolutional encoding. For detailed information refer to [2]. Moreover, the PHR is sent at the rate $850 \mathrm{~Kb} / \mathrm{s}$ to secure reliable reception whatever data rate follows it.

\begin{tabular}{|c|c|c|c|}
\hline \begin{tabular}{|l} 
SHR preamble \\
$16,64,1024,4096$ Symbols \\
$@ 850 \mathrm{~Kb} / \mathrm{s}$ \\
\end{tabular} & \begin{tabular}{|c|} 
SFD \\
8 symbols \\
$@ 850 \mathrm{~Kb} / \mathrm{s}$ \\
\end{tabular} & $\begin{array}{l}\text { PHR } \\
19 \text { Symbols } \\
\text { @ } 850 \mathrm{~Kb} / \mathrm{s}\end{array}$ & $\begin{array}{l}\text { Data field } \\
0-1209 \text { Symbols } \\
\text { @ } 850 \mathrm{~Kb} / \mathrm{s}, 6.81 \mathrm{Mb} / \mathrm{s}, 27.21 \mathrm{Mb} / \mathrm{s}\end{array}$ \\
\hline
\end{tabular}

Figure 2: Frame structure of IEEE802.15.4a

The VHDL coding for the transmitter starts building a frame according to the mode set by MAC. It generates firstly SHR by repeating preamble symbol by a given number. The sequence (code) structure (preamble symbol) and its number of repetition are configured over SPI from the MAC. The VHDL code for the complete baseband was developed and tested using the FPGA evaluation board ML605 REV E. The measurement and test setup are shown in Figure 3. The notebook is used here as a master for connecting it via SPI to the baseband. A Labview GUI was used for sending configuration commands from the master.

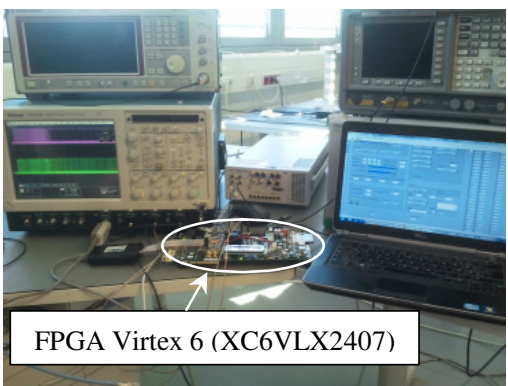

Figure 3: Measurement setup

The complete baseband was realized with 25398 Slice LUTs, 66 shift registers and 9925 slice registers. All timing constraints for operations running at both $31.2 \mathrm{MHz}$ and 499.2 $\mathrm{MHz}$ were met. An external clock of $31.2 \mathrm{MHz}$ was used as a reference and the two output signals OOK and BPSK generated from the transmitter were outputted through SMA connectors on the board for analysis.

Figure 4 shows OOK and BPSK bits delivered from the baseband to the analogue front end (AFE) at the rate of 499.2 $\mathrm{MHz}$. The waveform was generated from the oscilloscope Tektroniks. These bits are sent in two separate channels at the same time with the OOK bit informing presence of pulse and the BPSK indicating its polarity. The picture on the left side of the Figure 4 illustrates two preambles having a structure $\mathrm{S}=++00+00---+-0++-000+0+0-+0+0000$. As can be seen BPSK bits instruct the AFE transmitter to shift the phase of pulses by $180^{\circ}$. Generation of positive and negative pulses takes place then in the AFE.
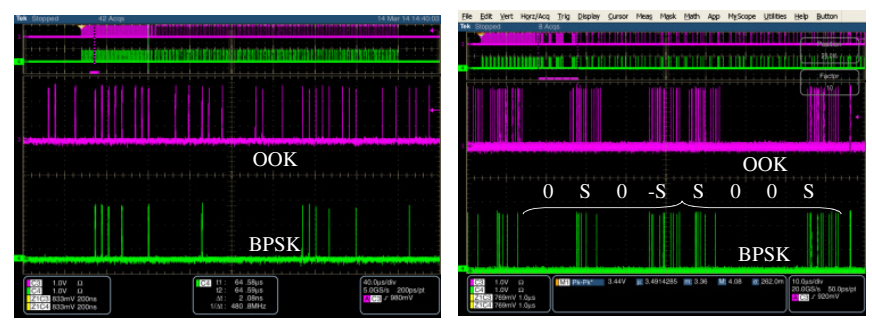

Figure 4: Preamble and SFD part of an IEEE802.15.4a frame

The right side of the Figure 4 shows the SFD field having a pattern 0S0-SSO0S built from the preamble S as suggested by the standard. A frame signal corresponding to the data rate 850 $\mathrm{Kb} / \mathrm{s}$ is visualized in Figure 5. The data field here is sent at 850 $\mathrm{Kb} / \mathrm{s}$ with an occurrence of a burst once around every $1 \mu \mathrm{s}$. The Figure 5 corresponds to differing lengths of SHR which is 16 and 64 .
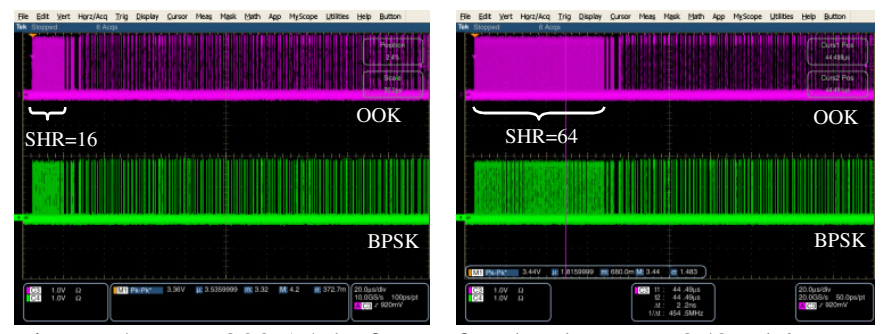

Figure 5: IEEE802.15.4a frame for the data rate $850 \mathrm{~Kb} / \mathrm{s}$ 
The frames captured for higher data rates $6.81 \mathrm{Mb} / \mathrm{s}$ and 27.24 $\mathrm{Mb} / \mathrm{s}$ appear in Figure 6. The PHR part is sent here at $850 \mathrm{~Kb} / \mathrm{s}$ and the payload at the rate stated being 8 and 16 times shorter than $850 \mathrm{~Kb} / \mathrm{s}$.

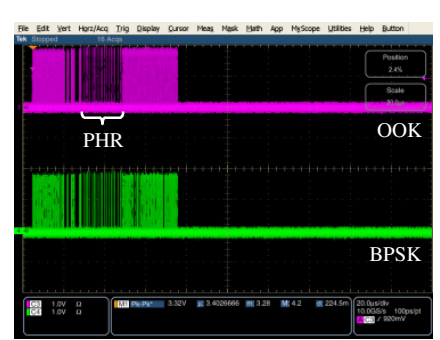

a) $6.81 \mathrm{Mb} / \mathrm{s}$

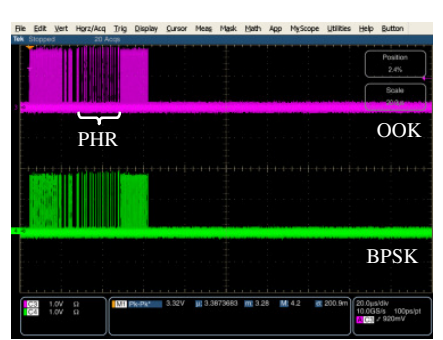

b) $27.24 \mathrm{Mb} / \mathrm{s}$
Figure 6: Frames generated for various data rates

It is worth to have a detailed look at bursts generated for various data rates. Figure 7 a) shows one burst for the data rate of $850 \mathrm{~Kb} / \mathrm{s}$. As can be seen, there are 16 OOK chips occupying 32 ns. Note that chips and pulses are used interchangeably. 9 chips out of these 16 have to be negative as indicated by the BPSK. For $6.81 \mathrm{Mb} / \mathrm{s}$, the burst has the duration of 4 ns as shown in Figure 7 b). The first pulse is negative and the second is positive. The task of the receiver is first to detect the presence of energy looking at the OOK and second is to decide phase of pulses constituting a burst.

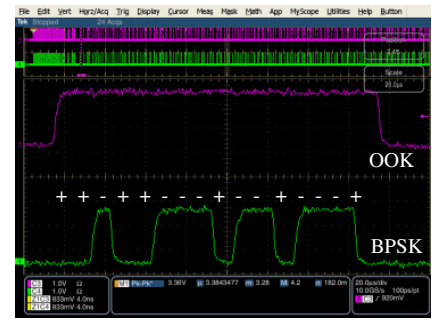

a) $850 \mathrm{~Kb} / \mathrm{s}$

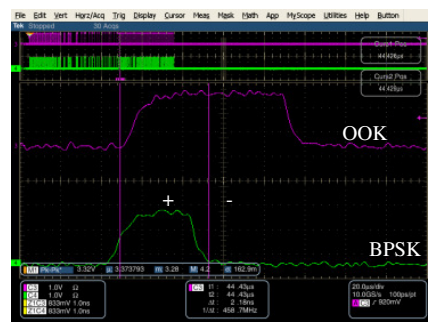

b) $6.81 \mathrm{Mb} / \mathrm{s}$
Figure 7: Burst structure for $850 \mathrm{~Kb} / \mathrm{s}$ and $6.81 \mathrm{Mb} / \mathrm{s}$

The burst of $27.24 \mathrm{Mb} / \mathrm{s}$ is depicted in Figure 8. It contains only one pulse with the duration of 2 ns and the phase of which being negative or positive carrying two bits: one is by the presence of pulse and the other one is by its polarity.

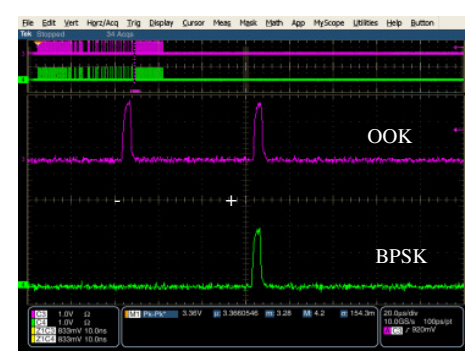

Figure 8: Burst of $27.24 \mathrm{Mb} / \mathrm{s}$

\section{IR-UWB RECEIVER}

The main task of the IR-UWB receiver is to detect signal in the presence of noise and other possible distortions. IR-UWB signal is characterized by its large bandwidth and low emission level being below $-41.3 \mathrm{dBm} / \mathrm{MHz}$. It also experiences large thermal noise due its high bandwidth which can lead to a short communication range especially at higher data rates. The noise power is calculated as follows:

$$
N_{0}=4 k B_{w} T=-87 \mathrm{dBm} \text {. }
$$

where $\mathrm{k}=4 * 1.38 * 10^{23} \mathrm{~J} / \mathrm{K}$ is the Bolzmann's constant, $\mathrm{T}=300 \mathrm{~K}$ denotes room temperature and $B_{w}$ is the bandwidth which is in our case $499.2 \mathrm{MHz}$.

For achieving a good error performance, the carrier to noise power ratio given as follows has to be high.

$$
\frac{C}{N}=\frac{E_{b}}{N_{0}} \frac{f_{b}}{B_{w}}
$$

where $C / N$ is the carrier to noise power ratio, $E_{b} / N_{0}$ denotes the energy per bit over noise power spectral density and $f_{b} /_{B_{w}}$ represents the data rate over the bandwidth. Different modulation schemes require certain $E_{b} /_{N_{0}}$ for guaranteeing needed bit error rate (BER). BPSK for instance requires it to be $8 \mathrm{~dB}$ for $\mathrm{BER}=10^{-4}$. Substituting other parameters such as $\mathrm{B}_{\mathrm{w}}=500 \mathrm{MHz}$ and $\mathrm{f}_{\mathrm{b}}=850 \mathrm{~Kb} / \mathrm{s}$ to the equation (2), the $\mathrm{C} / \mathrm{N}$ was found to be around $-20 \mathrm{dBm}$. If the signal is assumed to be attenuated by $60-80 \mathrm{~dB}$, which is reasonable for a range round $30 \mathrm{~m}$, it may go below noise level. Furthermore, due to its large thermal noise resulting from high bandwidth and its low emission power, IR-UWB can experience very weak receive signal. Therefore, it is necessary to test the receiver assuming noisy and distorted signal.

The AFE of the IR-UWB receiver amplifies and downconverts the signal to the baseband. The baseband was designed to receive I and Q signals in four channels each being 16 bits long as presented in the Figure 9. I and Q paths are used here to receive the signal experiencing clock deviation. A detailed consideration of such receiver is provided in [1].

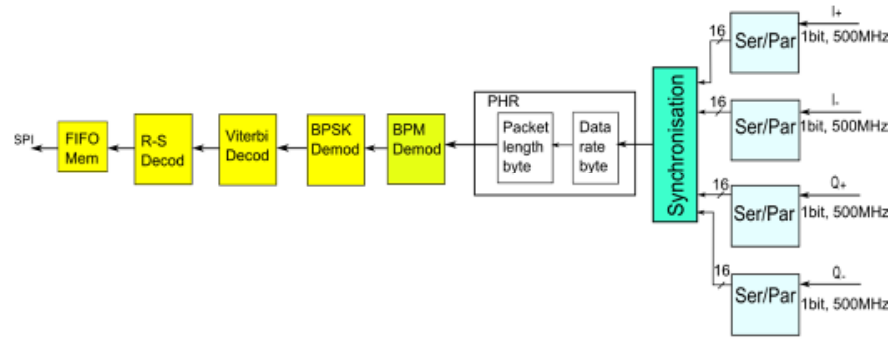

Figure 9: IR-UWB baseband receiver

The AFE performs digitization by using a comparator which compares the signal with a positive and negative threshold voltage having the same amplitude. The comparator outputs signal whenever the input signal goes higher in magnitude in both directions $\left(\mathrm{I}_{+}, \mathrm{I}_{-}\right)$. The output signal at the comparator was then level shifted to standard CMOS prior applying to the baseband. The same operation is performed at the Q path and therefore, the baseband receives in four channels 
simultaneously as it appears in Figure 9. The comparator output can be very short or long if the threshold is not set properly or if the input is distorted.

The baseband receiver starts by parallelizing the comparator output at $499.2 \mathrm{MHz}$ clock to 16 bits at $31.2 \mathrm{MHz}$ (See Figure 9). All the processing after the parallelization runs at $31.2 \mathrm{MHz}$ clock speed. The synchronization scheme adopted achieves 2 ns accuracy despite the slower clock by doing correlation for all 16 bit positions. It implies that 16 correlators search preamble for every clock cycle. As 15 zeros are inserted between the elements of the ternary sequence used for the preamble, one of the correlators can spot it delivering $2 \mathrm{~ns}$ accuracy. More detailed treatment of the synchronization algorithm can be found in [3].

Once the synchronization was established by detecting SFD field, PHY header is received which contains 19 bits including some redundancy. PHY header informs the receiver the data rate and the length of the packet to be received. The payload detection is only possible if the PHY header is received correctly. Therefore the PHR is sent always at $850 \mathrm{~Kb} / \mathrm{s}$ and protected differently against errors irrespective of the data rate than the payload. PHR bits are detected by looking at energy at the expected positions of the first and second half of symbols only. Decision for bit " 1 " is made if there is more energy in the first half and " 0 " if more energy is detected in the second half. The second bit carried with BPSK is detected by deciding if the phase of a sequence used for the burst is reversed or not. It can be said that position bits can be detected at lower data rates especially at $850 \mathrm{~Kb} / \mathrm{s}$ with high reliability as a large number of pulses is used transmitted forming a burst. However, detection of BPSK bits presents challenge as the phase of individual pulses has to be recovered. The phase detection is demanding since the pulse shaping, clock deviation and the digitization at the receiver have to be considered with great care. Once the data rate and the length of packet are known the payload detection proceeds in the same way as for the PHY header by evaluating energy level over the time interval corresponding to the data rate. For $6.81 \mathrm{Mb} / \mathrm{s}$ and $27.24 \mathrm{Mb} / \mathrm{s}$ the time interval to be examined for energy is $4 \mathrm{~ns}$ and $2 \mathrm{~ns}$ respectively. Higher data rates therefore require stricter synchronization for capturing a small number of pulses.

After the bits are decided with the demodulation described before, channel decoding with Viterbi and Reed Solomon is carried out. Data bits are always complete at all stages: output of the demodulator, output of Viterbi decoder as well as Reed Solomon decoder as the redundant bits introduced can be ignored. It is therefore possible to observe how much improvement is resulting through each stage of the detection.

\section{IR-UWB IMPLEMENTATION ISSUES}

As the process of manufacturing for an ASIC solution of the IR-UWB under discussion was chosen to be 250nm BiCMOS from IHP Germany, the clock frequency of $31.2 \mathrm{MHz}$ is adopted for the entire baseband operations except Par/Ser at the transmitter and Ser/Par at the receiver. For ensuring a least number of components to run at $499.2 \mathrm{MHz}$ which is convenient for the manufacturing, the parallelization and serialization are performed through four stages halving the clock 499.2 MHz at each step generating $31.2 \mathrm{MHz}$ at the last. By doing so, few logic gates needed for the clock division and multiplexing of 2 to 1 operate at the clock rate $499.2 \mathrm{MHz}$ reducing the risk of speed associated with the process. These converters were shown to be fully functional both in VHDL behavioral and layout simulation and ASIC chip testing.

At the receiver, down-converted $\mathrm{I}$ and $\mathrm{Q}$ signals with the bandwidth of $499.2 \mathrm{MHz}$ are made digital with a comparator which produces a pulse when the input signal exceeds the threshold set. Since the IR-UWB signal contains positive and negative pulses, four comparators are employed that work with positive and negative thresholds for I and Q signals. There is a possibility that short pulses at the comparator output may not be captured by the parallelizer if the threshold is not set properly or the comparator is not fast enough.

It is worth noting that the baseband was simulated extensively by comparing the VHDL behavioral as well as layout simulation results with noisy and faded IR-UWB frames generated by Matlab for various data rates and packet lengths. Furthermore, the receiver was tested applying the signal captured from signal generators (N8242A-arbitrary waveform generator for the baseband, E8267D-vector signal generator for the analogue high frequency frontend) that produce IR-UWB frames corresponding to the standard IEEE802.15.4a for all data rates and packet configuration.

\section{IR-UWB TESTING \& MEASUREMENT}

The performance of any communication system is evaluated by BER for certain signal to noise ratio (SNR). It is suitable to adopt this method when considering the analogue high frequency front end. Since our present consideration focuses on the baseband, the test was done differently. There is no relation on showing how the high frequency noisy and distorted signal can manifest itself in the digital baseband specific to the IRUWB. If there is a clock deviation between the transmitter and the receiver, receive signal may get distorted as shown in Figure 10 where signal experiences periodic phase shift and envelope distortion.

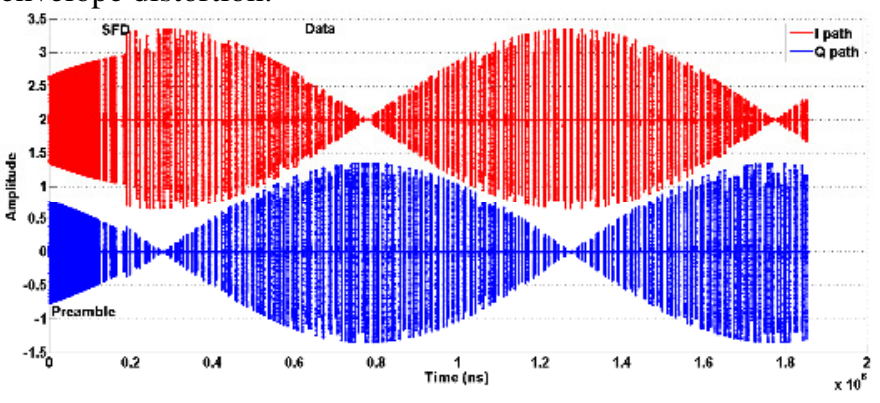

Figure 10. IR-UWB frame signal subjected to carrier frequency deviation of $16 \mathrm{KHz}$ at $7.9872 \mathrm{GHz}$. 
Such case can happen in reality as transmitter and receiver use always separate local reference clocks which can deviate from its central value and alter with time. Hence it has to be considered in the design in particular for the detection algorithm. We carried out measurements and tests in following ways. To demonstrate the performance of the baseband, we applied the baseband signal generated from the transmitter to a distorting block as shown in Figure 11.

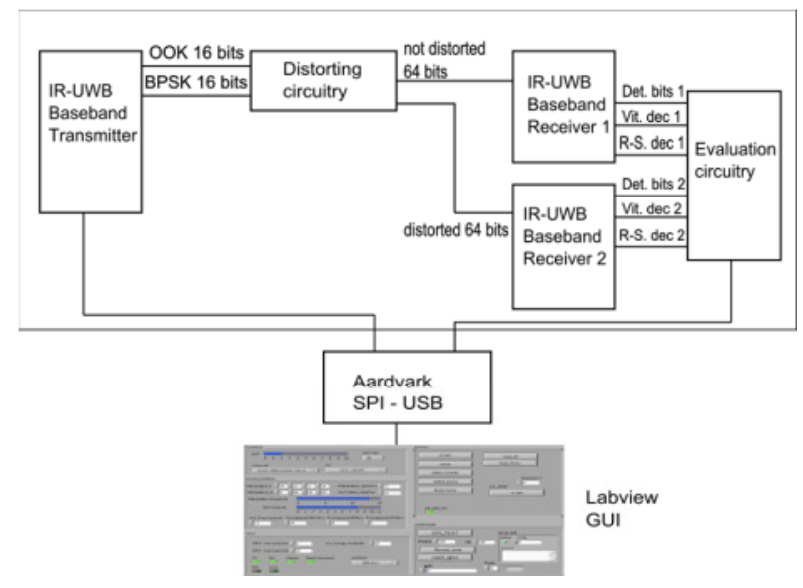

Figure 11. IR-UWB baseband measurement procedure

OOK and BPSK bits are distorted in various ways and then applied to the receiver. In other words $\mathrm{I}_{+}, \mathrm{I}_{-}, \mathrm{Q}_{+}, \mathrm{Q}_{\text {- signal are }}$ received firstly without any impairments and secondly with some distortion through the baseband receiver 1 and 2 respectively. The results are evaluated by comparing the outputs from the two receivers.

\section{A. Synchronisation performance}

Synchronization performance was measured by distorting SHR and SFD field various ways. The goal was to establish how many chip errors can the receiver tolerate. For this purpose the distorting block alters the signal at its output by setting it to zeros or ones for each specified period over given length of distortion in terms of clock cycles as demonstrated in Figure 12. The start of distortion was shifted by one cycle of the clock 31.2 $\mathrm{MHz}$ and the number of shift was chosen to be 100 covering around $3,2 \mu \mathrm{s}$ ( $\left.32 \mathrm{~ns}^{*} 100\right)$ to ensure that the bursts are affected by the distortion being introduced.

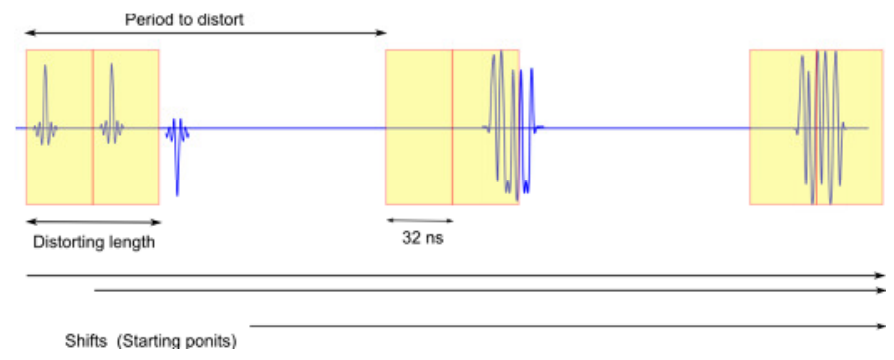

Figure 12. Introducing periodic distortion to IR-UWB frame for evaluating the performance of baseband receiver
Synchronization performance was measured by distorting the SHR and SFD parts of a frame in a way mentioned before. As the duration of a preamble symbol is $992 \mathrm{~ns}$ or in other word it is 31 cycles of the clock $31.2 \mathrm{MHz}$, we started distorting every 31 cycle of a frame by setting 16 bits in the cycle either all to zero or to ones as well as selectively. It was observed that up to five elements out of 31 can be distorted in the preamble signal whereby the receiver still can detect the preamble and synchronize. Note that the length of the distortion here stretches over up to 24 elements of a preamble. The distorting period was reduced down to 7 implying that in the worst case 24 elements of each preamble was distorted. This indicates that the adopted synchronization scheme can tolerate up to five errors. The performance started to degrade with distortion extending over 6 cycles (See Figure 13).

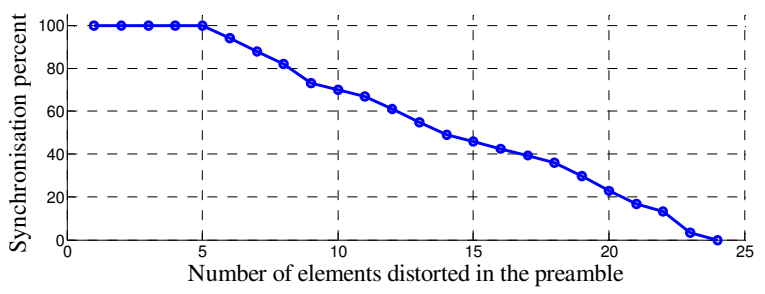

Figure 13. Synchronisation performance evaluated for 300 packets

The synchronization algorithm which does correlation and evaluates its result is discussed in [3]. The ASIC chip delivers a synchronization performance comparable to the simulation.

\section{B. Data detection performance}

The data detection performance was evaluated in the same manner as for the synchronization. The packets sent consist of 17 bytes (total 204 bits including PHY Header). The payload part of the packet was introduced a distortion with certain frequency lasting one cycle which is $32 \mathrm{~ns}$. The signal in this period was set to zero resembling a situation where there is no signal. The measurement results are represented in Figure 14. As can be seen, packet reception is $100 \%$ correct when the frequency of distortion is larger than $3.4 \mu$ s for a coded system where the Reed-Solomon decoding is integrated. It implies that every 3-4th symbols of the $850 \mathrm{~Kb} / \mathrm{s}$ frame was distorted. However, as the distortion is periodic and the position of bursts varies from symbol to symbol not all burst are affected. Since a part of packet is always distorted, such testing can still give a good idea on the detecting performance of the baseband implemented.

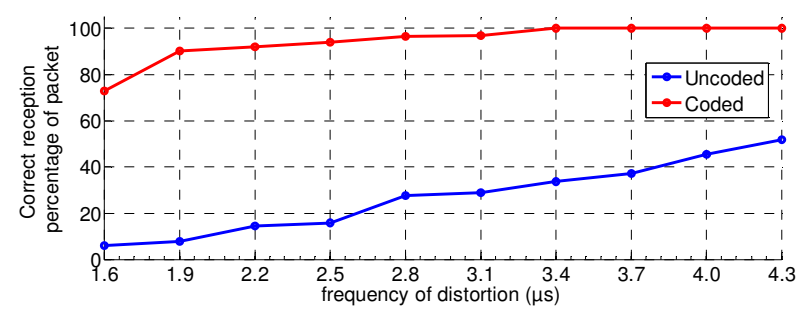

Figure 14. Packet reception performance for $850 \mathrm{~Kb} / \mathrm{s}$ under a periodic distortion extending over $32 \mathrm{~ns}$. 
A similar test was performed for higher data rates $6.81 \mathrm{Mb} / \mathrm{s}$ and $27.24 \mathrm{Mb} / \mathrm{s}$. In these cases we had to distort more frequently as the packets are 8 and 16 times shorter respectively in comparison to $850 \mathrm{~Kb} / \mathrm{s}$. For the $6.81 \mathrm{Mb} / \mathrm{s}$, the packets were received $100 \%$ correctly by correcting it for the distortion introduced every $352 \mathrm{~ns}$ occupying $4 \mathrm{~ns}$ which is one burst. Some initial tests carried out with the ASIC chip designed and fabricated as discussed in this work suggest that all the data rates can be supported. If clocks are synchronized and the threshold of the comparator is set appropriately, it can communicate wirelessly at all data rates. In particular, 850 $\mathrm{Kb} / \mathrm{s}$ was measured to be quite robust as a sufficient number of pulses are received as a burst. However, detecting the phase of individual pulses as well as burst still remains as a challenge due to the limited bandwidth implemented and the highest clocking speed of 499.2 MHz chosen. Despite being unable to recover phase causing the Viterbi decoder to turn off, the ASIC solution for IR-UWB can offer satisfactory error performance at the transmit data rates mentioned above.

\section{ASIC solution for the baseband}

The introduced baseband was made ready as an ASIC in 250 nm BiCMOS technology and integrated to the AFE. Its layout takes an area of $3.5 \mathrm{~mm} \times 1.7 \mathrm{~mm}$ as shown in Figure 15. The design passed all simulation and tests necessary for tape-out. Initial tests of the ASIC solution show that the complete baseband functions and the simulation and ASIC test results agree to a great degree. The power consumption of the baseband is approximately $74 \mathrm{~mW}$ at $2.85 \mathrm{~V}$ supply both by simulation and real tests for the entire design including extensive testing circuitries. The ASIC baseband can generate frames for all data rates according to the standard and receive them. Even higher data rates were tested to be operational especially at $27.24 \mathrm{Mb} / \mathrm{s}$ for wireless communication.

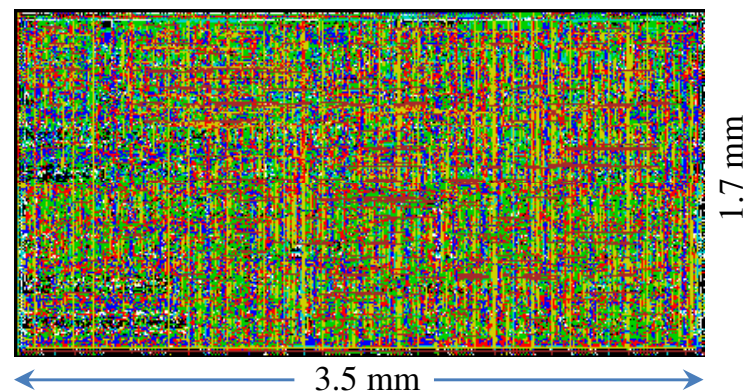

Figure 15. Layout of the IEEE.802.15.4a IR-UWB baseband

\section{CONCLUSION}

A configurable IR-UWB digital baseband that is conform to the standard IEEE802.15.4a was designed and implemented in FPGA as well as an ASIC. The baseband takes a small area in ASIC being only $3.5 \mathrm{~mm} \times 1.7 \mathrm{~mm}$. The transmitter generates all waveforms for the data rates $850 \mathrm{~Kb} / \mathrm{s}, 6.81 \mathrm{Mb} / \mathrm{s}$ and 27.24 $\mathrm{Mb} / \mathrm{s}$. The receiver was tested by introducing distortion to the signal being received. The synchronization performance was found to be $100 \%$ robust for the distortion that affects up to 6 elements of the preamble symbol. Packet detection is reliable for all data rates even under very frequent periodic distortion.

\section{ACKNOWLEDGMENT}

The research work and its results presented in this paper were carried out within the project KUSZ which is being funded by the German Ministry of Education and Research (BMBF). The authors wish to extend their gratitude to the KUSZ project consortium for their very productive and inspiring cooperation which leads to a remarkable outcome.

\section{REFERENCES}

[1] S. Olonbayar, D. Kreiser, R. Kraemer.; „Performance and implementation of multi-rate IR-UWB baseband transceiver for IEEE802.15.4a“, ICUWB2013, Sydney, Sep. 2013

[2] Standard specification IEEE802.15.4a, 2007

[3] S. Olonbayar, G. Fischer, R. Kraemer "Synchronisation performance of wireless sensor networks", ICUWB2008, pp. 59-62, no. 2 Hannover, Germany, Sep 2008

[4] S. Olonbayar, D. Kreiser, D. Martynenko, G. Fischer, O. Klymenko, R. Kraemer.; „Board implementation and its performance for IR-UWB IEEE.802.15.4a from multiple ASIC chips“, Invited paper, EW2012, Poznan, Poland, April 2012 